TRANSACTIONS OF THE

AMERICAN MATHEMATICAL SOCIETY

Volume 349, Number 11, November 1997, Pages 4401-4414

S $0002-9947(97) 01866-7$

\title{
ORDER EVALUATION OF PRODUCTS OF SUBSETS IN FINITE GROUPS AND ITS APPLICATIONS. II
}

\author{
Z. ARAD AND M. MUZYCHUK
}

\begin{abstract}
In this paper we give a new estimate of the cardinality of the product of subsets $A B$ in a finite non-abelian simple group, where $A$ is normal and $B$ is arbitrary. This estimate improves the one given in J. Algebra 182 (1996), 577-603.
\end{abstract}

\section{INTRODUCTION}

This paper is a continuation of [2], where the following question was considered. Given a finite group $G$ and two arbitrary subsets $S, T \subset G$, how large may their product $T S$ be, provided that $T S \neq G$ ?

In [2] the survey of related results was presented. In particular, we proved that if $S$ is a normal subset, $|S|>1$, and $G$ is finite non-abelian simple, then $S T \neq G$ yields that $|S T| \geq|S|+|T|-1$. Furthermore, the equality $|S T|=|S|+|T|-1$ holds if and only if either $|T|=1$ or $T=\bar{S}^{-1} \mathrm{~g}$, where $\bar{S}$ denotes the complement to $S$ in $G$.

As it was illustrated in [2], the above-mentioned result implies various interesting applications which were stated there.

The purpose of this paper is to present a better estimation for $|A B|$. More precisely, the main result of the paper is

Theorem 1.1. Let $G$ be a finite non-abelian simple group. Denote by $l$ the minimal cardinality of non-trivial conjugacy classes of $G$. Then for each normal $A \subset G$, such that $1<|A| \leq|G| / 4$ and for any $B \subset G$,

$$
|B| \geq 2, \quad|A B| \leq|G|-2 \Rightarrow|A B| \geq|A|+|B|+(l-18) / 12 .
$$

In particular, if $A$ is a non-trivial conjugacy class, then either $\left|C_{G}(a)\right|=3, a \in A$, or the assumption $|A| \leq|G| / 4$ holds by the simplicity of $G$. Non-abelian simple groups $G$ with self-centralizing subgroup of order 3 are $A_{5}$ and $P S L(2,7)$ by [5]. If $G=A_{5}$, then $l=12$ and Theorem 1.1 holds by [2]. If $G=P S L(2,7)$, then $l=21$ and $|A|=56$. Here also one can prove that Theorem 1.1 holds. Therefore, if $A$ is a non-trivial conjugacy class of $G$, then the assumption $|A| \leq|G| / 4$ may be omitted.

Received by the editors September 25, 1995.

1991 Mathematics Subject Classification. Primary 20D99, 05A99; Secondary 05C25.

This work was done at the Gelbart and Emmy Noether Research Institutes for Mathematical Sciences at Bar-Ilan University.

The second author was supported by the research grants from the Israeli Ministry of Science and the German-Israeli Foundation for fundamental research.

(C)1997 American Mathematical Society 
As an application of Theorem 1.1 we prove

Theorem 1.2. Let $G$ be a finite non-abelian simple group. Then for each normal $A \subset G$, such that $1<|A| \leq|G| / 4$ and for any $B \subset G$, it holds that

$$
|B| \geq 2,|A B| \leq|G|-2 \Rightarrow|A B| \geq|A|+|B|+3 \text {. }
$$

As a direct consequence we obtain the following omnibus theorem:

Theorem 1.3. Let $G$ be a finite non-abelian group with $k$ conjugacy classes and $\mathrm{Cla}(G)^{\#}$ be the set of its non-trivial conjugacy classes. Then $G$ is not simple if one of the following holds:

1) $C D \subseteq C \cup D$ for some $C, D \in C l a(G)^{\#}$;

2) $C D \subseteq C^{-1} \cup D$ for some $C, D \in C l a(G)^{\#}$;

3) $C D \subseteq C^{-1} \cup D^{-1}$ for some $C, D \in C l a(G)^{\#}$;

4) $\prod_{B \in \mathcal{B}} B \subseteq \bigcup_{B \in \mathcal{B}} B \cup\{1\}$ for some $\mathcal{B} \subset C l a(G)^{\#}$;

5) there exist $\mathcal{A}, \mathcal{B} \subset \mathrm{Cla}(G)^{\#}$ such that

$$
\begin{aligned}
& \prod_{A \in \mathcal{A}} A \subseteq \bigcup_{B \in \mathcal{B}} B \cup\{1\}, \\
& \prod_{B \in \mathcal{B}} B \subseteq \bigcup_{A \in \mathcal{A}} A \cup\{1\} .
\end{aligned}
$$

6) $C C^{-1} \subseteq C \cup C^{-1} \cup\{1\}$ for some $C \in C l a(G)^{\# \text {; }}$

7) $C^{2} \subseteq C \cup C^{-1}$ for some $C \in C l a(G)^{\#}$;

8) $C^{2} \subseteq\{1\} \cup D \cup D^{-1}$ for some $C, D \in C l a(G)^{\#}$;

9) $\prod_{C \in C l a(G)} C \neq G$;

10) $\left|\prod_{C \in C l a(G) \# \backslash\{D\}} C\right|<|G|-1$, where $D \in C l a(G)^{\#}$ is a conjugacy class of minimal cardinality;

11) if $|C| \geq|G| / k-2, k>6, C \in C l a(G)^{\#}$ and $C^{k} \neq G$.

Parts 1) and 2) are known by [1]. Parts 3)-5), 7)-9) and 11) were open problems; a few of them were mentioned in [1]. Part 6) was proved in [1] by using CFSG. Part 9) is known due to R. Brauer (see [4]). The detailed structure of $G$ satisfying part 1) is known by [1]. In [1] it was shown that there is no finite group satisfying part 2). ${ }^{1}$

Further research is needed for a better understanding of the structure of $G$ satisfying parts 3)-11).

\section{Preliminaries}

Let $A \subset G$ be a subset of a group $G$. In what follows we use $\bar{A}$ for $G \backslash A$. For an integer $i$ we define

$$
\begin{gathered}
\mathcal{S}_{i}(A)=\{B \subset G|| B \mid>i \text { and }|\overline{A B}|>i\} \\
\omega_{i}(A)=\min \left\{|A B|-|B| \mid B \in \mathcal{S}_{i}(A)\right\} \\
\mathcal{E}_{i}(A)=\left\{B \in \mathcal{S}_{i}(A)|| A B|=| B \mid+\omega_{i}(A)\right\} .
\end{gathered}
$$

Since $\mathcal{S}_{i}(A) \subseteq \mathcal{S}_{j}(A)$ when $i \leq j, \omega_{i}(A)$ is a non-decreasing function of $i$.

Proposition 2.1. Let $X, Y \in \mathcal{E}_{i}(A)$ and $|X \cap Y|>i,|\overline{A X \cup A Y}|>i$. Then $X \cap$ $Y, X \cup Y \in \mathcal{E}_{i}(A)$.

\footnotetext{
${ }^{1}$ Character theorems dual to parts 7) and 8) were considered in [7].
} 
Proof. The identity

$$
|A X \cup A Y|+|A X \cap A Y|=|A X|+|A Y|
$$

implies

$$
|A(X \cup Y)|+|A(X \cap Y)| \leq|A X|+|A Y|=|X|+|Y|+2 \omega_{i}(A) .
$$

The inequalities $|X \cap Y|>i,|\overline{A X \cup A Y}|>i$ guarantee that $X \cap Y, X \cup Y \in \mathcal{S}_{i}(A)$. Therefore,

$$
|A(X \cup Y)|+|A(X \cap Y)| \geq|X \cup Y|+|X \cap Y|+2 \omega_{i}(A)=|X|+|Y|+2 \omega_{i}(A) .
$$

Combining this with (1) yields

$$
\begin{aligned}
& |A(X \cup Y)|=|X \cup Y|+\omega_{i}(A), \\
& |A(X \cap Y)|=|X \cap Y|+\omega_{i}(A),
\end{aligned}
$$

as claimed. $\diamond$

Proposition 2.2. (i) $\omega_{i}(A)=\omega_{i}\left(A^{-1}\right)$;

(ii) $B \in \mathcal{E}_{i}(A) \Rightarrow A^{-1}(\overline{A B})=\bar{B}$ and, consequently, $\overline{A B} \in \mathcal{E}_{i}\left(A^{-1}\right)$;

(iii) $B \in \mathcal{E}_{i}(A) \Leftrightarrow B g \in \mathcal{E}_{i}(A)$ for each $g \in G$;

(iv) if $A$ is normal, then

$$
\begin{gathered}
B \in \mathcal{E}_{i}(A) \Rightarrow A\left(\overline{A B}^{-1}\right)=\bar{B}^{-1}, \quad \text { and, consequently, } \overline{A B}^{-1} \in \mathcal{E}_{i}(A), \\
B \in \mathcal{E}_{i}(A) \Leftrightarrow g B h \in \mathcal{E}_{i}(A) \text { for any } g, h \in G .
\end{gathered}
$$

Proof. (i) It is sufficient to show that $\omega_{i}\left(A^{-1}\right) \leq \omega_{i}(A)$. Take an arbitrary $B \in$ $\mathcal{E}_{i}(A)$. Then $|A B|=|B|+\omega_{i}(A)$. If $g \in \overline{A B}$, then $A^{-1} g \cap B=\emptyset$, implying $A^{-1}(\overline{A B}) \subset \bar{B}$. Thus $|\overline{A B}|>i<|B| \leq\left|\overline{A^{-1}(\overline{A B})}\right|$. Therefore $\overline{A B} \in \mathcal{S}_{i}\left(A^{-1}\right)$, which implies

$$
\begin{aligned}
|\bar{B}| & \geq\left|A^{-1}(\overline{A B})\right| \geq \omega_{i}\left(A^{-1}\right)+|\overline{A B}|=\omega_{i}\left(A^{-1}\right)+|G|-|A B| \\
& =\omega_{i}\left(A^{-1}\right)+|G|-|B|-\omega_{i}(A)=\omega_{i}\left(A^{-1}\right)+|\bar{B}|-\omega_{i}(A) .
\end{aligned}
$$

(ii) Since $\omega_{i}(A)=\omega_{i}\left(A^{-1}\right)$, the inequality (2) implies

$$
|G|-|B| \geq\left|A^{-1}(\overline{A B})\right| \geq|G|-|B| .
$$

Therefore, $\left|A^{-1}(\overline{A B})\right|=|\bar{B}|$. Combining this with an inclusion $A^{-1}(\overline{A B}) \subset \bar{B}$ yields $A^{-1}(\overline{A B})=\bar{B}$. Now the inclusion $\overline{A B} \in \mathcal{E}_{i}\left(A^{-1}\right)$ easily follows from the following sequence of equalities:

$$
\begin{aligned}
\left|A^{-1}(\overline{A B})\right| & =|\bar{B}|=|G|-|B|=|A B|+|\overline{A B}|-|B| \\
& =\omega_{i}(A)+|\overline{A B}|=\omega_{i}\left(A^{-1}\right)+|\overline{A B}| .
\end{aligned}
$$

Proof of (iii) is a trivial exercise. Part (iv) is a direct consequence of (ii)-(iii) and normality of $A$. 


\section{Estimation of $\omega_{1}(A)$ of a normal subset $A \subset G$}

In what follows, we assume that $A \subseteq G, A \neq G$ is normal and $\mathcal{S}_{1}(A) \neq \emptyset$. It is easy to see that $\mathcal{S}_{1}(A) \neq \emptyset$ if and only if there exists $b \in G^{\#}$ with $|A\{1, b\}| \leq|G|-2$. Denoting by $m(A)$ the minimal value of $|A g \cup A|-|A|, g \in G^{\#}$, we can say that $\mathcal{S}_{1}(A) \neq \emptyset$ if and only if $m(A)+|A| \leq|G|-2$. Since $m(A) \leq|A|$, the latter inequality always holds in the case of $2|A|+2 \leq|G|$. If $m(A)=0$, then a subgroup $\operatorname{Sta}(A)=\{g \in G \mid g A=A\}$ is a non-trivial proper normal subgroup of $G$. The parameter $m(A)$ gives us an upper bound for $\omega_{1}(A)$. Indeed, $|A\{1, b\}| \geq \omega_{1}(A)+2$ whenever $1 \neq b$ and $|A\{1, b\}| \leq|G|-2$. Therefore

$$
m(A)-2 \geq \omega_{1}(A)-|A| .
$$

Moreover the equality case in (3) holds if and only if $\mathcal{E}_{1}(A)$ contains a subset with two elements.

In this section we study the situation where $\mathcal{E}_{1}(A)$ contains no 2-element subset, or, equivalently, $m(A)-2>\omega_{1}(A)-|A|$.

The main result may be formulated as follows:

Theorem 3.1. Let $A \subset G$ be a normal subset of a finite group $G$ with $\mathcal{S}_{1}(A) \neq \emptyset$ and $\omega_{1}(A)-|A|<m(A)-2$. Let $B \in \mathcal{E}_{1}(A)$ be of minimal cardinality such that $1 \in B$. If $|B|>\omega_{1}(A)-|A|+3$, then $B$ is a subgroup of $G$ such that $\left[G: N_{G}(B)\right] \leq 2$.

As a direct consequence, we obtain the following two results.

Theorem 3.2. Let $A \subset G$ be a normal subset such that $\mathcal{S}_{1}(A) \neq \emptyset$. Assume that $\omega_{1}(A)-|A|<(m(A)-3) / 2$. Then there exists a proper subgroup $H<G$ such that $\left[G: N_{G}(H)\right] \leq 2$ and $|A H|=\omega_{1}(A)+|H|$.

Theorem 3.3. Let $G$ be a non-abelian finite simple group. Let $A \subset G$ be an arbitrary normal subset of $G$ such that $\mathcal{S}_{1}(A) \neq \emptyset$. Then

$$
|B| \geq 2, \quad|G|-2 \geq|A B| \Rightarrow|A B| \geq|A|+|B|+\frac{m(A)-3}{2}
$$

holds for any $B \subset G$.

The rest of this section contains the proof of Theorem 3.1. Thus we always assume that $\mathcal{S}_{1}(A) \neq \emptyset$ and $m(A)-2>\omega_{1}(A)-|A|$. The following notation will be used throughout the section:

- $k:=\omega_{1}(A)-|A|$

- $B \in \mathcal{E}_{1}(A)$ is of minimal cardinality, $m:=|B|, m>2$;

- $C:=\overline{A B}, n:=|C|$.

We always have

$$
|G|=\omega_{1}(A)+m+n \Leftrightarrow|G|=|A|+k+m+n .
$$

According to Proposition 2.2 (iv), $C^{-1} \in \mathcal{E}_{1}(A)$. Therefore $n \geq m \geq 3$.

Lemma 3.1. Let $B_{1}, B_{2} \in \mathcal{E}_{1}(A)$ and $\left|B_{1}\right|=\left|B_{2}\right|=m$. Write $A B_{i}=\bar{C}_{i}, i=1,2$. Then

(i) $\left|B_{1} \cap B_{2}\right| \in\{0,1, m\}$;

(ii) either $\left|B_{1} \cap C_{2}^{-1}\right|=\left|B_{2} \cap C_{1}^{-1}\right|=m$, or $\left|B_{1} \cap C_{2}^{-1}\right| \leq 1 \geq\left|B_{2} \cap C_{1}^{-1}\right|$. 
Proof. (i) Assume the contrary, i.e., $1<\left|B_{1} \cap B_{2}\right|<m$. Then

$$
\begin{aligned}
\left|A\left(B_{1} \cup B_{2}\right)\right|+\left|A\left(B_{1} \cap B_{2}\right)\right| & \leq\left|A B_{1} \cup A B_{2}\right|+\left|A B_{1} \cap A B_{2}\right| \\
& =\left|A B_{1}\right|+\left|A B_{2}\right|=2 \omega_{1}(A)+2|B| .
\end{aligned}
$$

Since $\left|B_{1} \cap B_{2}\right|>1,\left|A\left(B_{1} \cap B_{2}\right)\right| \geq \omega_{1}(A)+\left|B_{1} \cap B_{2}\right|$, implying

$$
\begin{aligned}
& \left|A\left(B_{1} \cup B_{2}\right)\right| \leq 2 \omega_{1}(A)+2|B|-\left|A\left(B_{1} \cap B_{2}\right)\right| \\
& \quad \leq \omega_{1}(A)+2|B|-\left|B_{1} \cap B_{2}\right| \leq \omega_{1}(A)+2 m-2 \leq \omega_{1}(A)+m+n-2=|G|-2 .
\end{aligned}
$$

Thus, $\left|B_{1} \cap B_{2}\right|>1<\left|\overline{A\left(B_{1} \cup B_{2}\right)}\right|$, and, by Proposition 2.1, $B_{1} \cap B_{2} \in \mathcal{E}_{1}(A)$ contrary to a minimality of $B$.

(ii) Assume that at least one of the inequalities

$$
\begin{gathered}
\left|B_{1} \cap C_{2}^{-1}\right| \leq 1, \\
\left|B_{2} \cap C_{1}^{-1}\right| \leq 1
\end{gathered}
$$

does not hold. WLOG $\left|B_{1} \cap C_{2}^{-1}\right|>1$. Since $B_{1} \in \mathcal{S}_{1}(A)$ and $\left|B_{1} \cap C_{2}^{-1}\right|>1$, $B_{1} \cap C_{2}^{-1} \in \mathcal{S}_{1}(A)$, which, in turn, implies

$$
\left|A\left(B_{1} \cap C_{2}^{-1}\right)\right| \geq \omega_{1}(A)+\left|B_{1} \cap C_{2}^{-1}\right| .
$$

On the other hand,

$$
\left|A\left(B_{1} \cap C_{2}^{-1}\right)\right| \leq\left|A B_{1} \cap A C_{2}^{-1}\right|=\left|A B_{1}\right|+\left|A C_{2}^{-1}\right|-\left|A\left(B_{1} \cup C_{2}^{-1}\right)\right| .
$$

Since $A C_{i}^{-1}=\bar{B}_{i}^{-1}, i=1,2$, the right part of the above inequality may be rewritten as follows:

$$
\begin{aligned}
\left|A B_{1}\right| & +\left|A C_{2}^{-1}\right|-\left|A\left(B_{1} \cup C_{2}^{-1}\right)\right| \\
& =\omega_{1}(A)+\left|B_{1}\right|+\omega_{1}(A)+\left|C_{2}\right|-\left|\bar{C}_{1} \cup \bar{B}_{2}^{-1}\right| \\
& =|G|+\omega_{1}(A)-\left|\overline{C_{1} \cap B_{2}^{-1}}\right| \\
& =\omega_{1}(A)+\left|C_{1} \cap B_{2}^{-1}\right| .
\end{aligned}
$$

Comparing (5) and (6) gives us

$$
1<\left|B_{1} \cap C_{2}^{-1}\right| \leq\left|C_{1} \cap B_{2}^{-1}\right|=\left|B_{2} \cap C_{1}^{-1}\right| .
$$

Applying the same arguments to $B_{2} \cap C_{1}^{-1}$, we obtain the inverse inequality which yields

$$
\left|B_{1} \cap C_{2}^{-1}\right|=\left|B_{2} \cap C_{1}^{-1}\right|>1 \text {. }
$$

Now we have

$$
\left|A C_{1}^{-1} \cup A B_{2}\right|=\left|\bar{B}_{1}^{-1} \cup \bar{C}_{2}\right|=\left|\overline{B_{1}^{-1} \cap C_{2}}\right|=|G|-\left|B_{1}^{-1} \cap C_{2}\right| \leq|G|-2 .
$$

Thus $\left|C_{1}^{-1} \cap B_{2}\right|>1<\left|\overline{A\left(C_{1}^{-1} \cap B_{2}\right)}\right|$, whence, by Proposition 2.1, $C_{1}^{-1} \cap B_{2} \in$ $\mathcal{E}_{1}(A)$.

Since $B_{2}$ has a minimal cardinality among the elements of $\mathcal{E}_{1}(A),\left|C_{1}^{-1} \cap B_{2}\right|=$ $\left|B_{2}\right|$, thus finishing the proof.

Corollary 3.2. Let $B \in \mathcal{E}_{1}(A)$ with $|B|=m$. Then

(i) for any $x, y \in G,|B \cap x B y| \in\{0,1,|B|\}$;

(ii) if $1 \in B$, then either $B$ is a subgroup of $G$ or $|g B \cap B| \leq 1 \geq|B g \cap B|$ holds for each $g \in G$. 
Proof. (i) is a direct consequence of the previous claim and Proposition 2.2, part (ii).

(ii) Assume that $|B g \cap B|>1$ for some $g \in G \backslash\{1\}$ (the case when $|g B \cap B|>1$ is considered analogously). Then $B g \in \mathcal{E}_{1}(A)$ and by Lemma $3.1|B g \cap B|=|B|$, or, equivalently, $B g=B$. Thus $B$ is a union of the left cosets of the cyclic subgroup $\langle g\rangle$. This implies that $x B \cap B$ is a union of the left $\langle g\rangle$-cosets as well. In particular, $|x B \cap B|$ is divisible by the order $o(g)$ of $g$. On the other hand, $|x B \cap B| \in\{0,1,|B|\}$ for all $x \in G$. Therefore $|x B \cap B| \in\{0,|B|\}$ for an arbitrary $x \in G$. That means $x B \cap B$ is either $\emptyset$ or $B$. Since $1 \in B, B$ is a subgroup of $G$.

The latter statement makes it reasonable to split the general case into two subcases, depending on whether $B$ is a subgroup or not.

3.1. $B$ is not a subgroup of $G$. In this section we show that, under the assumptions of Theorem 3.1, $B$ should be a subgroup of $G$. In fact, we prove a stronger result.

Lemma 3.3. If $B$ is not a subgroup of $G$ and $1 \in B$, then

$$
\frac{m(m-3)}{2} \leq k
$$

Write $A B=\bar{C}$, where $|B|=m,|C|=n$. For every $c \in C$ we have

$$
A B=\bar{C}, \quad A B c^{-1}=\overline{C c^{-1}} .
$$

By applying Lemma 3.1, part (ii), we obtain that either

$$
\left|B \cap\left(C c^{-1}\right)^{-1}\right|=\left|B c^{-1} \cap C^{-1}\right|=|B|,
$$

or

$$
\left|B \cap\left(C c^{-1}\right)^{-1}\right| \leq 1 \geq\left|B c^{-1} \cap C^{-1}\right| .
$$

Since $1 \in B$ and $c \in C$, either

$$
C^{-1} c \supset B \subset c C^{-1}
$$

or

$$
C^{-1} c \cap B=B \cap c C^{-1}=\{1\} .
$$

Let $C_{1}$ be a set of those $c \in C$ satisfying (8) and $C_{2}$ be a set of those $c \in C$ satisfying (7). Clearly $C=C_{1} \cup C_{2}$ and $C_{1} \cap C_{2}=\emptyset$.

Proposition 3.4. $\left|C_{1}\right| \geq m-1$.

Proof. Assume the contrary, i.e. $\left|C_{1}\right| \leq m-2$. Then $\left|C_{2}\right|=|C|-\left|C_{1}\right|=n-\left|C_{1}\right| \geq$ 2.

As follows from (7)

$$
B^{-1} C_{2} \subset C \supset C_{2} B^{-1} .
$$

This yields $C b \supset C_{2}$ for each $b \in B$, whence

$$
|A B B|=\left|\bigcup_{b \in B} A B b\right|=\left|\bigcup_{b \in B} \overline{C b}\right|=\left|\overline{\bigcap_{b \in B} C b}\right| \leq\left|\overline{C_{2}}\right| \leq|G|-2 .
$$

Therefore $B^{2} \in \mathcal{S}_{1}(A)$, whence

$$
\left|A B^{2}\right| \geq \omega_{1}(A)+\left|B^{2}\right| .
$$


On the other hand,

$$
\begin{gathered}
\left|A B^{2}\right| \leq\left|\bar{C}_{2}\right|=|G|-|C|+\left|C_{1}\right| \\
=|G|-n+\left|C_{1}\right| \leq|G|-n+m-2=\omega_{1}(A)+2 m-2 .
\end{gathered}
$$

Thus

$$
\omega_{1}(A)+\left|B^{2}\right| \leq \omega_{1}(A)+2 m-2,
$$

whence

$$
\left|B^{2}\right| \leq 2|B|-2 .
$$

But now $\left|B^{2}\right| \geq|B \cup B b|=2|B|-1$ yields a contradiction ( $B$ is not a subgroup, so $b \neq 1 \Rightarrow|B \cup B b|=2|B|-1)$. $\quad \diamond$

Proof of Lemma 3.3. We have two equalities:

$$
\begin{gathered}
A B c^{-1}=\overline{C c^{-1}}, \quad c \in C, \\
A C^{-1}=\overline{B^{-1}} .
\end{gathered}
$$

Therefore,

$$
\begin{aligned}
A\left(C^{-1} \cup B C_{1}^{-1}\right) & =A C^{-1} \cup\left(\bigcup_{c \in C_{1}} A B c^{-1}\right)=\overline{B^{-1}} \cup\left(\bigcup_{c \in C_{1}} \overline{C c^{-1}}\right) \\
& =\overline{B^{-1} \cap\left(\bigcap_{c \in C_{1}} C c^{-1}\right)} \subset G \backslash\{1\} .
\end{aligned}
$$

This implies

$$
B C_{1}^{-1} \cup C^{-1} \subset \overline{A^{-1}}
$$

whence

$$
\left|B C_{1}^{-1} \cup C^{-1}\right| \leq|G|-|A|=k+m+n .
$$

By definition of $C_{1}$ :

$$
B c^{-1} \cap C^{-1}=\left\{c^{-1}\right\}
$$

for all $c \in C_{1}$. Hence

$$
\left|B C_{1}^{-1} \cup C^{-1}\right|=\left|B^{\#} C_{1}^{-1} \cup C^{-1}\right|=\left|B^{\#} C_{1}^{-1}\right|+\left|C^{-1}\right|
$$

(here $B^{\#}=B \backslash\{1\}$ ). Together with (10) this yields

$$
\left|B^{\#} C_{1}^{-1}\right| \leq k+m \text {. }
$$

$B$ is not a subgroup; therefore, by Corollary $3.2,\left|B^{\#} c^{\prime} \cap B^{\#} c^{\prime \prime}\right| \leq 1$ whenever $c^{\prime} \neq c^{\prime \prime}$. Since $\left|C_{1}\right| \geq|B|-1=m-1$, we have at least $m-1$ sets $B^{\#} c, c \in C_{1}^{-1}$ of cardinality $m-1$ such that any pair of them has at most one element in common. This implies that $\left|B^{\#} C_{1}^{-1}\right|$ has at least $m(m-1) / 2$ elements. Together with (11), this implies $m(m-1) / 2 \leq k+m . \quad \diamond$ 
3.2. The case of $B$ being a subgroup of $G$. Denote $l=\left[G: N_{G}(B)\right]$. If $l \leq 2$, then we are done. Thus we may assume that $l \geq 3$. Let $B_{1}=B, B_{2}, \ldots, B_{l}$ be a complete set of conjugates to $B$.

$$
A B_{i}=\bar{C}_{i}, \quad A C_{i}^{-1}=\overline{B_{i}^{-1}}, \quad i=1, \ldots, l .
$$

By Lemma 3.1, $B_{i} \cap B_{j}=\{1\}$ whenever $i \neq j$. In other words, $B$ should be a TI-subgroup of $G$. Each $C_{i}$ is a union of $B_{i}$-cosets; therefore $m \mid n$. To prove Theorem 3.1 we consider two separate cases:

(i) $\left|C_{i} \cap C_{j}\right| \leq 1$ for each $i \neq j$.

(ii) there exists a pair $i \neq j$ with $\left|C_{i} \cap C_{j}\right| \geq 2$.

The first case is settled below.

Proposition 3.5. Case (i) is impossible.

Proof. We have $A C_{i}^{-1}=\overline{B_{i}^{-1}}, i=1,2, \ldots, l$. Therefore,

$$
A\left(C_{1}^{-1} \cup C_{2}^{-1} \cup C_{3}^{-1}\right) \subset G \backslash\{1\} .
$$

This implies

$$
C_{1}^{-1} \cup C_{2}^{-1} \cup C_{3}^{-1} \subset \overline{A^{-1}}
$$

whence

$$
3 n-3 \leq\left|C_{1}^{-1} \cup C_{2}^{-1} \cup C_{3}^{-1}\right| \leq|G|-|A|=k+m+n .
$$

Since $m \leq n$, we obtain $m \leq k+3$, a contradiction.

To consider the second case, we may assume that $\left|C_{1} \cap C_{2}\right| \geq 2$.

Denote $D=C_{1} \cap C_{2}$. For each $d \in D$ we can write

$$
A B_{1} d^{-1}=\overline{C_{1} d^{-1}}
$$

$$
A B_{2}=\bar{C}_{2}
$$

By Lemma 3.1, part (ii), either

$$
B_{1} d^{-1} \subset C_{2}^{-1} \text { and } B_{2} \subset\left(C_{1} d^{-1}\right)^{-1},
$$

or

$$
\left|B_{1} d^{-1} \cap C_{2}^{-1}\right| \leq 1 \geq\left|B_{2} \subset\left(C_{1} d^{-1}\right)^{-1}\right| .
$$

Equivalently, either

$$
d B_{1} \subset C_{2} \text { and } B_{2} d \subset C_{1},
$$

or

$$
B_{1} \cap C_{2}^{-1} d=B_{2} \cap d C_{1}^{-1}=\{1\}
$$

Now Theorem 3.1 is a direct consequence of the following claim.

Lemma 3.6. If $\left|C_{1} \cap C_{2}\right| \geq 2$, then $m \leq k+2$.

Proof. First assume that there exist at least two elements $d_{1}, d_{2} \in D$ which satisfy (16), i.e.,

$$
B_{1} \cap C_{2}^{-1} d_{i}=B_{2} \cap d_{i} C_{1}^{-1}=\{1\}, \quad i=1,2 .
$$


Then we have three equalities

$$
\begin{aligned}
& A B_{1} d_{1}^{-1}=\overline{C_{1} d_{1}^{-1}} \subset G \backslash\{1\}, \\
& A B_{1} d_{2}^{-1}=\overline{C_{1} d_{2}^{-1}} \subset G \backslash\{1\}, \\
& A C_{2}^{-1}=\overline{B_{2}^{-1}} \subset G \backslash\{1\} .
\end{aligned}
$$

Now $A\left(B_{1} d_{1}^{-1} \cup B_{1} d_{2}^{-1} \cup C_{2}^{-1}\right) \subset G \backslash\{1\}$, whence $B_{1} d_{1}^{-1} \cup B_{1} d_{2}^{-1} \cup C_{2}^{-1} \subset \overline{A^{-1}}$. This gives us the following inequality

$$
\left|B_{1} d_{1}^{-1} \cup B_{1} d_{2}^{-1} \cup C_{2}^{-1}\right| \leq|G|-|A|=\omega_{1}(A)-|A|+m+n=k+m+n .
$$

By (17) the left side may be estimated as follows: ${ }^{2}$

$$
\left|B_{1} d_{1}^{-1} \cup B_{1} d_{2}^{-1} \cup C_{2}^{-1}\right|=2\left|B_{1}\right|-2+|C|=2 m+n-2 .
$$

Hence $2 m+n-2 \leq k+m+n$, as required.

Thus we may assume that the number of elements of $D$ satisfying (16) is not greater than 1. Therefore, there is a subset $F \subset D$ such that $|F| \geq|D|-1$ and

$$
f B_{1} \subset C_{2}, \quad B_{2} f \subset C_{1}
$$

holds for all $f \in F$.

We claim that $F B_{1}=F$. Indeed, $f B_{1} \subset C_{2}$ for each $f \in F$. On the other hand, $f \in C_{1}$ and $C_{1} B_{1}=C_{1}$, implying $f B_{1} \subset C_{1}$. Therefore, $f B_{1} \subset C_{1} \cap C_{2}=D$ This shows that an element $f b, b \in B_{1}$ doesn't satisfy (16) for each $b \in B_{1}$. Hence $f b$ satisfies (15), whence $f b \in F$.

Write

$$
\begin{gathered}
\left|A B_{2} B_{1}\right|=\left|\left(A B_{1} \cup A B_{2}\right) B_{1}\right|=\left|\left(\bar{C}_{1} \cup \bar{C}_{2}\right) B_{1}\right|=\left|\bar{D} B_{1}\right| \\
\quad=\left|\bigcup_{b \in B_{1}} \overline{D b}\right| \leq|\bar{F}|=|G|-|F| \leq|G|-|D|+1 .
\end{gathered}
$$

Since $F B_{1}=F$ and $F \neq \emptyset,|F| \geq\left|B_{1}\right|=m$. Hence $\left|A B_{2} B_{1}\right| \leq|G|-2$ and we can write

$$
\left|A B_{2} B_{1}\right| \geq \omega_{1}(A)+\left|B_{2} B_{1}\right|=\omega_{1}(A)+|B|^{2}=\omega_{1}(A)+m^{2} .
$$

Thus

$$
\begin{aligned}
\omega_{1}(A)+m^{2} & \leq|G|+1-|D|=|G|-\left|C_{1} \cap C_{2}\right|+1=\left|\overline{C_{1} \cap C_{2}}\right|+1 \\
& =\left|\bar{C}_{1} \cup \bar{C}_{2}\right|+1=\left|A B_{1} \cup A B_{2}\right|+1 \\
& \leq 2 \omega_{1}(A)+2|B|-|A|+1=2 \omega_{1}(A)-|A|+2 m+1 .
\end{aligned}
$$

Finally,

$$
m^{2}-2 m \leq \omega_{1}(A)-|A|+1=k+1 .
$$

Since $m \geq 3, m \leq m^{2}-2 m<k+2$ as desired.

Proof of Theorem 3.2. Let $B \in \mathcal{E}_{1}(A)$ be of minimal cardinality $m$. WLOG $1 \in B$. Since $\omega_{1}(A)<(m(A)-3) / 2+|A|<m(A)-2+|A|, m>2$. If $|B|>\omega_{1}(A)-|A|+3$, then we have completed our proof via Theorem 3.1. Otherwise, $|B| \leq \omega_{1}(A)-|A|+3$ and $|A B|=|A|+|B|+k \leq|A|+2 k+3$. But $|B|>2$. Therefore $|A B| \geq|A|+m(A)$. Consequently, $2 k+3 \geq m(A)$, contrary to our assumption

$$
\omega_{1}(A)-|A|=k<\frac{m(A)-3}{2} .
$$

This is a contradiction.

\footnotetext{
${ }^{2}$ Since $B_{1} d_{i}^{-1} \cap C_{2}^{-1}=\left\{d_{i}^{-1}\right\}$ and $d_{1} \neq d_{2}, B_{1} d_{1}^{-1}$ and $B_{1} d_{2}^{-1}$ are disjoint $B_{1}$-cosets.
} 


\section{The estimation of $m(A)$}

In this section we assume that $G$ is a finite non-abelian simple group with a normal subset $A,|A| \leq|G| / 4$.

For each $\lambda \geq 0$ we define

$$
A_{\lambda}=\{g \in G|| A \cup A g|\leq| A \mid+\lambda\}=\{g \in G|| A \cap A g|\geq| A \mid-\lambda\} .
$$

Clearly, $A_{\lambda}$ is a normal subset of $G$ and $A_{\lambda} \subset A_{\mu}$ whenever $\lambda \leq \mu$. Further, $A_{\lambda}=G$ for each $\lambda \geq|A|$. The simple calculations give us

$$
\sum_{g \in G \backslash\{1\}}|A \cap A g|=|A|^{2}-|A| .
$$

Lemma 4.1. $A_{\lambda} A_{\mu} \subset A_{\lambda+\mu}$.

Proof. Take an arbitrary $g \in A_{\lambda}$ and $h \in A_{\mu}$. One can write

$$
\begin{gathered}
|A \cup A h g|=\left|A g^{-1} \cup A h\right| \leq\left|A g^{-1} \cup A h \cup A\right| \\
=\left|\left(A g^{-1} \cup A\right) \cup(A h \cup A)\right|=\left|A g^{-1} \cup A\right|+|A h \cup A|-\left|\left(A g^{-1} \cup A\right) \cap(A h \cup A)\right| \\
\leq|A|+\lambda+|A|+\mu-|A|=|A|+\lambda+\mu . \quad \diamond
\end{gathered}
$$

Since $1 \in A_{\lambda}$ for each $\lambda \geq 0$, then $\left|A_{\lambda}\right| \geq 1$ for all $\lambda \geq 0$. As follows from the definition, $m(A)$ is the minimal $\lambda$ with $\left|A_{\lambda}\right|>1$. We abbreviate $m:=m(A)$. Since $G$ is simple, $0<m$. In what follows we write $F_{n}=A_{n m} \backslash A_{m(n-1)}, n \geq 1$. In particular, $F_{1}=A_{m} \backslash\{1\}$. It is clear that $F_{n}, n \geq 1$ are disjoint and $A_{n m}=\{1\} \cup F_{1} \cup \ldots \cup F_{n}$.

Lemma 4.2. If $A_{m n} \neq G$ for some $n \geq 2$, then

(i) $\quad\left|A_{m n}\right| \geq\left|F_{1}\right|+\left|A_{m(n-1)}\right|$;

(ii) $\quad\left|F_{n}\right| \geq\left|F_{1}\right|$;

(iii) $\quad\left|A_{n m}\right| \geq 1+n\left|F_{1}\right|$.

Proof. (i) Since $G$ is simple, the implication

$$
|A B| \neq|G| \Rightarrow|A B| \geq|A|+|B|-1
$$

holds for each pair $A, B$ of normal subsets (see Theorem 1.4 of [2]).

By Lemma $4.1 A_{m} A_{m(n-1)} \subset A_{n m} \neq G$, whence

$$
\left|A_{n m}\right| \geq\left|A_{m}\right|+\left|A_{m(n-1)}\right|-1=\left|F_{1}\right|+\left|A_{m(n-1)}\right| .
$$

(ii) Since $A_{n m} \supset A_{m(n-1)},\left|F_{n}\right|=\left|A_{n m}\right|-\left|A_{m(n-1)}\right|$ and (ii) follows.

Part (iii) of the claim follows from (i) and (ii). $\diamond$

Lemma 4.3. If $\left|F_{1}\right| \geq|A|$, then $3 m>|A|$.

Proof. At first consider the case $A_{2 m}=G$. Since $|A \cap A g| \geq|A|-\lambda$ for all $g \in A_{\lambda}$, the inequality $|A \cap A g| \geq|A|-2 m$ holds for all $g \in G$. By applying (20) we obtain

$$
|A|(|A|-1) \geq(|A|-2 m)(|G|-1)>(|A|-2 m) \cdot 3|A| .
$$

After cancellation we obtain

$$
|A|-1>3|A|-6 m
$$

and the claim follows. 
Assume now that $A_{2 m} \neq G$. Then $\{1\} \cup F_{1} \cup F_{2} \neq G$ and, due to (20),

$$
|A|(|A|-1) \geq \sum_{g \in F_{1}}|A \cap A g|+\sum_{g \in F_{2}}|A \cap A g| \geq(|A|-m)\left|F_{1}\right|+(|A|-2 m)\left|F_{2}\right| .
$$

But $\left|F_{2}\right| \geq\left|F_{1}\right| \geq|A|$ by Lemma 4.2. Therefore $|A|(|A|-1) \geq(2|A|-3 m)|A|$. This completes the proof.

Let us order the elements of $G=\left\{g_{0}=1, \ldots, g_{n-1}\right\}, n=|G|$, in such a way that $i<j$ implies $\lambda_{i} \leq \lambda_{j}$, where $\lambda_{j}=\left|A \cap A g_{j}\right|$.

Proposition 4.4. If $j \leq\left|F_{1}\right| i$, then $\lambda_{j} \geq|A|-m i$.

Proof. We claim that $j \leq\left|F_{1}\right| i$ implies that $g_{j} \in A_{m i}$. Indeed, this inclusion is evident in the case $A_{m i}=G$. Thus, we can assume that $A_{m i} \neq G$, which implies, according to (23), that $\left|A_{m i}\right| \geq 1+i\left|F_{1}\right|$. Therefore, $A_{m i}$ contains $m\left|F_{1}\right|+1$ first elements of $G$, i.e., $g_{j} \in A_{m i}$ for each $0 \leq j \leq i\left|F_{1}\right|$. As follows from the definition of $A_{m i}, \lambda_{j}=\left|A \cap A g_{j}\right| \geq|A|-m i$.

Proposition 4.5. Let $n$ be an integer satisfying

$$
\frac{2|A|}{3 m} \leq n \leq \frac{2|A|}{3 m}+1
$$

and $\left|F_{1}\right| \leq|A|$. Then $n\left|F_{1}\right| \leq|G|-3$.

Proof. Denote $a=|A|$. Since $\left|F_{1}\right| \leq|A|$ and $|G| \geq 4|A|$, it is sufficient to show that $\left|F_{1}\right|(n-1) \leq 3 a-3$. Assume the contrary, i.e. $\left|F_{1}\right|(n-1) \geq 3 a-2$. Then, by Proposition $4.4, \lambda_{3 a-2} \geq a-(n-1) m$, whence

$$
\lambda_{3 a-2} \geq a-(n-1) m \geq a-\frac{2 a}{3 m} m=\frac{a}{3} .
$$

Therefore, $\lambda_{i} \geq a / 3$ for all $1 \leq i \leq 3 a-2$. But this implies that $a(a-1) \geq$ $a(3 a-2) / 3$, which is a contradiction

Theorem 4.1. At least one of two inequalities

$$
\left|F_{1}\right|<3 m, \quad|A|<6 m
$$

holds.

Proof. Assume the contrary, i.e. $\left|F_{1}\right| \geq 3 m$ and $|A| \geq 6 m$. By Lemma 4.3, $\left|F_{1}\right|<|A|$. Take an integer $n$ such that ${ }^{3}$

$$
\frac{2 a}{3 m} \leq n \leq \frac{2 a}{3 m}+1
$$

Due to Proposition 4.5, $n\left|F_{1}\right| \leq|G|-3$. Consider the sets $S_{i}=\left\{g_{j}|i| F_{1} \mid \geq j>\right.$ $\left.(i-1)\left|F_{1}\right|\right\}, i=1, \ldots, n$. Clearly $\left|S_{j}\right|=\left|F_{1}\right|$. Since $n\left|F_{1}\right| \leq|G|-3, S_{1} \cup \ldots \cup S_{n} \subset$ $G \backslash\{e\}$. By Proposition $4.4 \lambda_{j} \geq a-m i$ for all $j$ satisfying $g_{j} \in S_{i}$. Therefore,

$$
a(a-1) \geq \sum_{i=1}^{n}(a-m i)\left|S_{i}\right|=\left|F_{1}\right|\left(n a-m \frac{n(n+1)}{2}\right) \geq 3 m\left(n a-m \frac{n(n+1)}{2}\right) .
$$

By the choice of $n, m \geq \frac{2 a}{3 n}$, whence

$$
a(a-1) \geq 3 \cdot \frac{2 a}{3 n}\left(n a-m \frac{n(n+1)}{2}\right)=2 a^{2}-a m(n+1) .
$$

\footnotetext{
${ }^{3}$ Here, as before, $a=|A|$.
} 
After simple transformations, we obtain $m(n+1) \geq a+1$. On the other hand, $n+1 \leq \frac{2 a}{3 m}+2$, whence

$$
\left(\frac{2 a}{3 m}+2\right) m \geq a+1 \Leftrightarrow \frac{2 a}{3}+2 m \geq a+1 \Leftrightarrow 2 m \geq \frac{a}{3}+1
$$

contrary to $m \leq a / 6$. $\diamond$

As a corollary we obtain the following:

Theorem 4.2. Let $A$ be a normal subset of $G$ with $|A| \leq|G| / 4$. Denote by $l$ the cardinality of the smallest non-trivial conjugacy class of $G$. Then

$$
m(A)>\min (l / 3,|A| / 6) \geq l / 6 .
$$

Proof. Due to Theorem 4.1, $m(A)=m>\left|F_{1}\right| / 3$ or $m(A)=m>|A| / 6$. But $F_{1}$ is a non-trivial normal set. Therefore $m(A)>l / 3$ or $m(A)>|A| / 6$, as desired. $\quad \diamond$

It is easy to see that Theorem 1.1 is a direct consequence of this result and of Theorem 3.3.

\section{Proofs of Theorems $1.2,1.3$}

Proof of Theorem 1.2. Denote by $l$ the minimal cardinality of non-trivial conjugacy classes of $G$. If $l \geq 43$, then Theorem 1.1 implies our claim. Thus we may assume that $l \leq 42$ which implies that $G$ has a primitive permutation representation of a degree of 42 at most. The classification of all primitive groups of a degree of 50 at most, was done in [8] without CFSG. According to [3], either $G=A_{n}$ or a point stabilizer of $G$ has a trivial centre. Thus, in the case of $G \neq A_{n}, G$ has a maximal subgroup of index of, at most, 21. Due to [3], $G$ is one of the following groups given in Table 1.

\section{TABLE 1}

\begin{tabular}{|l|l|}
\hline$G$ & degree \\
\hline$A_{5}$ & 6 \\
$A_{6}$ & 10 \\
$L_{2}(8)$ & 9 \\
$L_{2}(16)$ & 17 \\
$L_{2}(7)$ & 7 \\
$L_{2}(11)$ & 11 \\
$L_{2}(13)$ & 14 \\
$L_{2}(17)$ & 18 \\
$L_{2}(19)$ & 20 \\
$L_{3}(3)$ & 13 \\
$M_{11}$ & 11 \\
$M_{12}$ & 12 \\
$A_{n}$ & $n \leq 42$ \\
\hline
\end{tabular}

The groups $A_{n}, n \geq 7, L_{3}(3), M_{11}, M_{12}$ have no non-trivial conjugacy class with fewer than 43 elements.

The groups $L_{2}(p)$, $p$ odd, $p>7, L_{2}(8), L_{2}(16)$ have no non-trivial conjugacy class with fewer than 40 elements according to 8.27 of [6]. 
In the case of $G=A_{6}$, there are only two normal subsets $A$ of $G$ satisfying the assumption $|A| \leq|G| / 4$, namely: the conjugacy classes $C_{1}$ and $C_{2}$ of cyclic types $[3]$ and [3,3], respectively. Using the multiplication tables of the conjugacy classes of $A_{6}$, one can easily check that $m(A) \geq 8$ in both cases, $A=C_{1}$ and $A=C_{2}$. Therefore, by Theorem 3.3,

$$
|A B| \geq|A|+|B|+(m(A)-3) / 2>|A|+|B|+2,
$$

as desired.

The case of $G=L_{2}(7)$ may be settled analogously.

Consider now the remaining case $G=A_{5}$. Denote by $C_{1}, C_{2}, C_{3}, C_{4}$ all its nontrivial conjugacy classes (we assume that $\left|C_{1}\right|=\left|C_{2}\right|=12,\left|C_{3}\right|=15,\left|C_{4}\right|=20$ ). There are only three normal subsets $A$ of $A_{5}$ satisfying $|A| \leq|G| / 4: A=C_{1}, A=$ $C_{2}, A=C_{3}$. If $A=C_{3}$, then $m(A) \geq 8$ and we are done. Since $C_{1}$ and $C_{2}$ are conjugate by an outer automorphism of $A_{5}$, it is enough to consider the only case of $A=C_{1}$. In this case, $m(A)=7$ and the arguments we used before do not work. To show that our claim remains true even in this case, we assume the contrary, i.e.

$$
\exists B \subset G, \quad|B|>1 \text { and }|G|-2 \geq|A B| \leq|A|+|B|+2 .
$$

We also assume that $B$ has a minimal cardinality among all subsets of $A_{5}$ satisfying the above conditions.

If $B$ is not a subgroup, then by Lemma $3.3|B|(|B|-3) / 2 \leq \omega_{1}(A)-|A| \leq 2$. Therefore $|B| \leq 4$, whence $|A B| \leq|A|+|B|+2 \leq|A|+6$. On the other hand, $|B| \geq 2$ implies that $|A B| \geq|A|+m(A)=|A|+7$. This is a contradiction. Hence $B$ should be a subgroup of $A_{5}$. By Theorem $3.1|B| \leq 3+\omega_{1}(A)-|A| \leq 5$. But direct calculations show that $|A B| \geq|A|+|B|+3$ for each subgroup $B \leq A_{5},|B| \leq 5$. $\diamond$

As a direct consequence we obtain the proof of Theorem 1.3.

1)-4) and 6)-9) are immediate corollaries of Theorem 4.2.

5) If $G$ is simple, then

$$
\begin{aligned}
& 3+\sum_{A \in \mathcal{A}}|A| \leq\left|\prod_{A \in \mathcal{A}} A\right| \leq 1+\sum_{B \in \mathcal{B}}|B| ; \\
& 3+\sum_{B \in \mathcal{B}}|B| \leq\left|\prod_{B \in \mathcal{B}} B\right| \leq 1+\sum_{A \in \mathcal{A}}|A|,
\end{aligned}
$$

a contradiction.

10) Assume that $G$ is simple and $C l a(G)^{\#}=\left\{C_{1}, \ldots, C_{k}\right\}$ with $\left|C_{1}\right| \leq\left|C_{2}\right| \leq$ $\ldots \leq\left|C_{k}\right|$.

Consider $C_{2} \cdot \ldots \cdot C_{k}$. We claim that $\left|C_{2} \cdot \ldots \cdot C_{k}\right| \geq|G|-1$. Indeed, if it is not true, then by Theorem $1.2\left|C_{2} \cdot \ldots \cdot C_{k}\right| \geq\left|C_{2}\right|+\ldots+\left|C_{k}\right|+3$, implying $\left|C_{2} \cdot \ldots \cdot C_{k}\right| \geq\left|C_{2}\right|+\ldots+\left|C_{k}\right|+\left|C_{1}\right|=|G|-1$. Again, a contradiction.

Thus $\left|C_{2} \cdot \ldots \cdot C_{k}\right| \geq|G|-1$.

11) If $\left|C^{k}\right| \leq|G|-2$, then $|G|-2 \geq\left|C^{k}\right| \geq k|C|+3(k-1) \geq|G|+k-3$, implying $k \leq 1$, a contradiction. Thus $\left|C^{k}\right|=|G|-1$ is the unique case we have to consider. In this case, $C^{k}=G \backslash\{1\}$, which, in turn, implies $C^{k-1} \subset \overline{C^{-1}}$. Hence

$$
|C|(k-1)+3(k-2) \leq\left|C^{k-1}\right| \leq|G|-|C| .
$$

Consequently, $|C| k+3 k-6 \leq|G| \leq k|C|+2 k$. Whence $k \leq 6$, contrary to the assumption. 


\section{REFERENCES}

[1] Z.Arad, H.Blau, On table algebras and their applications to finite group theory, J. Algebra, 138 (1991), 137-185. MR 92f:20007

[2] Z.Arad, E.Fisman, M.Muzychuk. Order evaluation of products of subsets in finite groups and its applications.I, J. Algebra 182 (1996), 577-603. CMP 96:15

[3] I.D.Dixon, B. Mortimer. The primitive permutation groups of degree less than 1000, Math. Proc. Camb. Phil. Soc., 103 (1988), 213-238. MR 89b:20014

[4] W.Feit. Characters of Finite Groups, Benjamin, New York, Amsterdam, 1967. MR 36:2715

[5] W.Feit and J.Thompson. Finite groups which contain a self-centralizing subgroup of order 3. Nagoya Math. J. 21, (1962), pp. 185-197. MR 26:192

[6] B.Huppert, N.Blackburn, Finite groups II, Springer-Verlag, 1982. MR 84i:20001a

[7] I.M.Isaacs and Ilan Zisser. Squares of characters with a few constituents in finite groups. Arch. Math. 63, 1994, pp. 197-207. MR 95e:20015

[8] B.A.Pogorelov, Primitive groups of permutations of small degrees. II, Algebra i Logika, 19 1980, n.4, pp. 423-457. MR 82j:20009b

Department of Mathematics \& Computer Science, Bar-Ilan University, 52900 RamatGAN, ISRAEL 